\title{
Newly developed semiconductor detectors by Hamamatsu
}

\section{Koei Yamamoto ${ }^{1}$, Kazuhisa Yamamura, Kenichi Sato, Shogo Kamakura,}

\section{Tsuyoshi Ota, Hiroki Suzuki}

Solid State Division, Hamamatsu Photonics K.K.

Hamamatsu-City, 435-8558 Japan

\section{Shinji Ohsuka}

Central Research Laboratory, Hamamatsu Photonics K.K.

Hamamatsu-City, 434-8601 Japan

\begin{abstract}
We developed the novel photon counting device named Multi-Pixel Photon Counter (MPPC). It is a solid state photon counter using Geiger-mode APD and self-quenching resistor. It has many features like low bias voltage operation, high gain, insensitive to magnetic field and mechanical robustness. The MPPC is one of the products of Si-PM (Silicon Photomultiplier) family, which was originally developed in Russia. HAMAMATSU MPPC is designed as a photon counting device based on structures of a Si APD named "HPK Reverse Structure", which has been adopted by CERN (CMS calorimeter).
\end{abstract}

International workshop on new photon-detectors PD07

Kobe University, Kobe, Japan

27-29 June, 2007

\footnotetext{
$1 \quad$ Speaker
} 


\section{Introduction}

In the many application fields like academic, medical, measurement and industrial, the demands for counting the number of photons with high accuracy are increasing. MPPC can be a candidate device to fulfill such a requirement. It has many features like high gain $\left(10^{5}\right.$ to $\left.10^{6}\right)$, low dark count rate $(<1 \mathrm{MHz}$ with 0.5 p.e. threshold level), low bias voltage operation $(<100 \mathrm{~V})$, insensitive to magnetic fields, room temperature operation, high Photon Detection Efficiency (PDE), high time resolution, low power consumption and mechanical robustness.

Some application fields need to couple MPPC with scintillator. We can know incident photons or particle energy, timing and even position by catching scintillation light. Many popular scintillators have peak of the emission of light wavelength around $400 \mathrm{~nm}$. To achieve high accuracy measurement, MPPC is controlled to have high Photon Detection Efficiency (PDE) around 400nm by adopting the well selected structure. We call this structure as "HPK Reverse Structure”. [1] [2] [3] [4]

\section{Mechanism of MPPC}

\subsection{Geiger-mode APD and quenching resistor}

MPPC is composed by the array of components which consist of Geiger- mode operating APD (G-APD) and quenching resistor. Geiger-mode is achieved by biasing the voltage higher than breakdown voltage by a few volts. In this operating mode, avalanche process will occur with certain probability when the excited charge was generated. In general, G-APD itself does not have the capability to stop the avalanche process. It will be continued till the electric field became weak enough to end the avalanche process. The quenching resistor plays the role to control the quantity of inflow charge to G-APD. The inflow charge to the G-APD can be seen as the recharge process of G-APD. The lack of inflow charge causes the voltage down of G-APD and avalanche process will be stop at certain voltage. The too big quenching resistor value causes long recovery time, which is determined by RC time constant, and the too small value causes the non quenching process. It is important to control the quenching resistor value matching with each pixel type, structure and application. [5] [6]

\subsection{Dynamic range and PDE}

Each pixel of MPPC performs as "digital” counter. It can’t count how many photons were incident into the pixel at the same time. It only knows whether incidence of photon had happened or not. MPPC has the saturation limit for number of photons incidence in the same time. It is necessary to prepare the enough pixels for many incidental photons. PDE of MPPC is lead from equation below.

$$
P D E=F_{\text {geom }} \times \text { Q.E. } \times P_{\text {avalanche }}
$$


$F_{\text {geom }}$ is geometry factor, Q.E. is quantum efficiency and $P_{\text {avalanche }}$ is avalanche probability. As for the MPPC, quenching resistor and circuit are theoretically necessary for each pixel. It causes the decrease of active area for large number of pixel type MPPC. On the other hand, small pixel type MPPC has enough space, and it can be achieved large PDE by widening active area. But the dynamic range will become small. There is a trade-off between dynamic range and PDE. Hamamatsu has six kinds of MPPC lineups from the viewpoint of dynamic range and package as below.

$\begin{array}{ccll}\text { Type No. } & \text { Number of Pixels } & F_{\text {geom }} & \text { Pixel Pitch } \\ \text { S10362-11-025C/U } & 1600 & 30.8 \% & 25 \mathrm{um} \\ \text { S10362-11-050C/U } & 400 & 61.5 \% & 50 \mathrm{um} \\ \text { S10362-11-100C/U } & 100 & 78.5 \% & 100 \mathrm{um}\end{array}$

The last index means CERAMIC type package (C) and CAN type package (U).

\subsection{Photon absorption coefficient}

Fig. 1 shows the photon absorption coefficient and absorption length of silicon as a function of wavelength. S10362-11 series is optimized to 400nm wavelength. So, the absorption length is determined almost uniformly (green arrow on the Fig. 2 is the main target range of MPPC). [7]

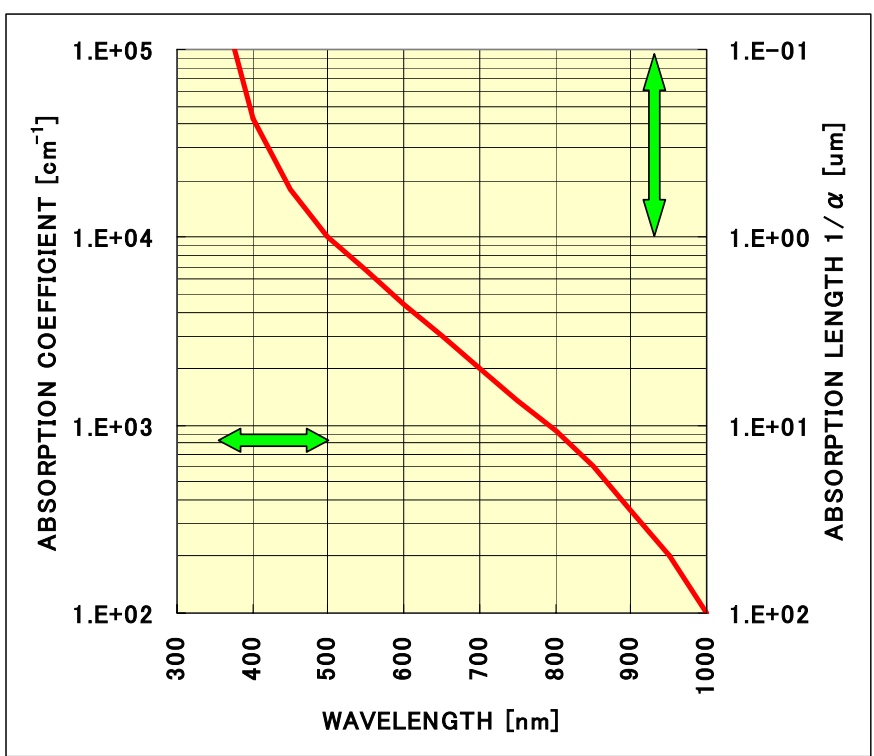

Fig. 1: Photon Absorption coefficient of Silicon

\section{$2.4 \quad$ Impact ionization coefficient}

The number of electron-hole pairs created by carrier running in unit length is called impact ionization coefficient. This coefficient is depending upon whether electric carriers that run are electrons or holes. Impact ionization coefficient of electron and hole is represented as $\alpha$ and 
$\beta$ respectively. Fig. 2 shows the impact ionization coefficient of silicon as a function of the electric field. The electric field strength of Geiger operating MPPC reaches to several $10^{5} \mathrm{~V} \mathrm{~cm}^{-1}$ (green arrow range in Fig. 2) and $\alpha$ is always higher than $\beta$. The first ionizing carrier determines the probability of avalanche occurrence. To achieve high PDE, electron injection to the avalanche region is necessary and MPPC selected p-type silicon to window area. [8]

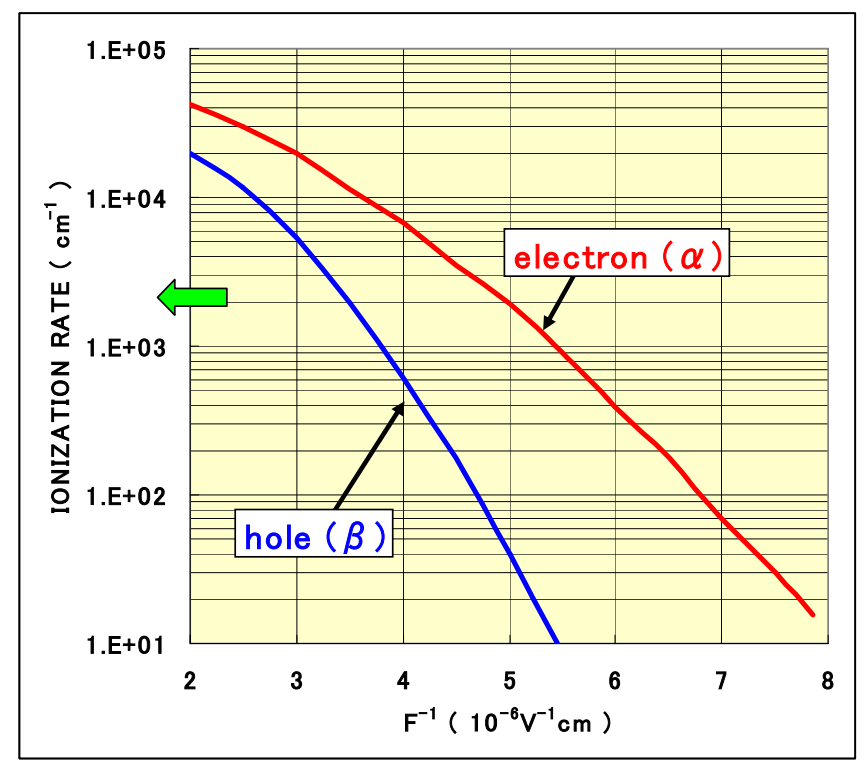

Fig. 2: Impact ionization coefficient as a function of electric field

\subsection{Structure}

The structure of MPPC is based on the Hamamatsu S8664-55 APD (Fig. 3). S8664-55 has been adopted as electromagnetic calorimeter of CMS experiment.

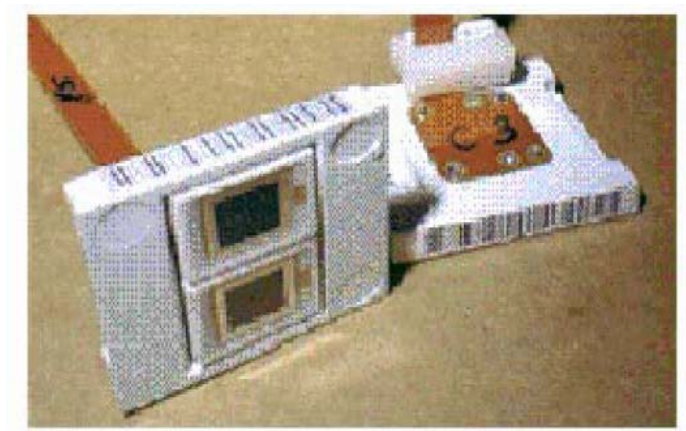

Fig. 3: Hamamatsu S8664-55 APD (mounted on CMS jig)

Fig. 4 shows the S8664-55 structure and Electric field strength. The $\mathrm{p} / \mathrm{n}$ junction is formed in an epitaxial layer. The operation voltage is 350 to $400 \mathrm{~V}(\mathrm{M}=50)$. Fig. 5, 6 show Q.E. and gain uniformity respectively. 

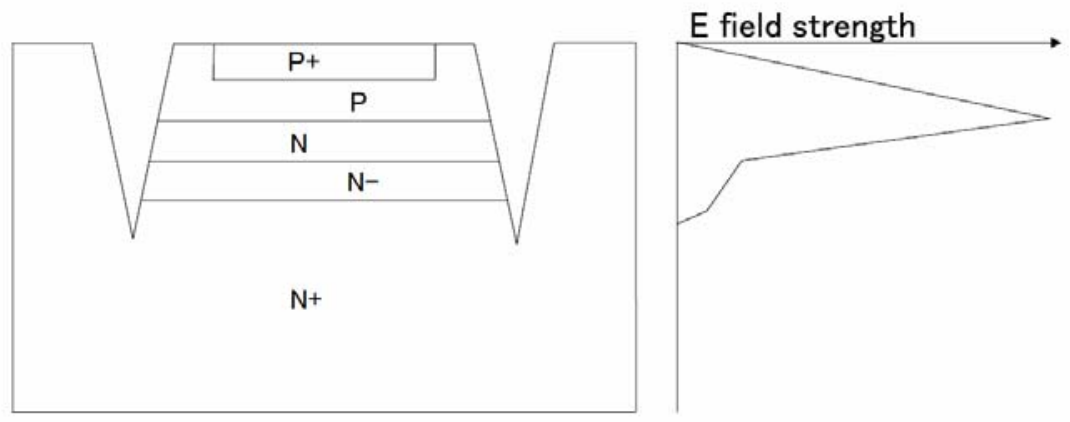

Fig. 4: Hamamatsu Reverse Structure APD for CMS

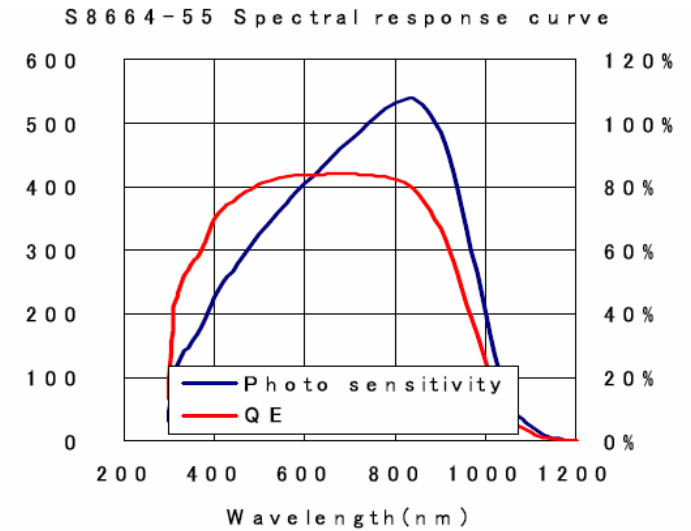

Fig. 5: Q.E of S8664-55

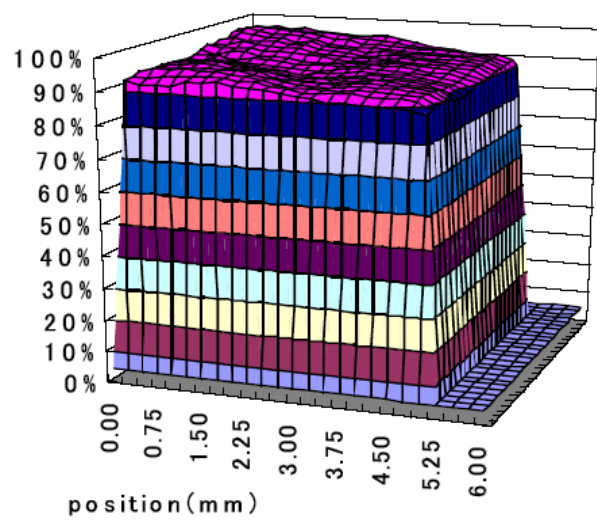

Fig. 6: Gain uniformity of S8664-55

Based on this S8664-55 APD, we carefully chose the structure named "HPK Reverse structure”. Fig. 7 shows the process of selection of structure of MPPC.

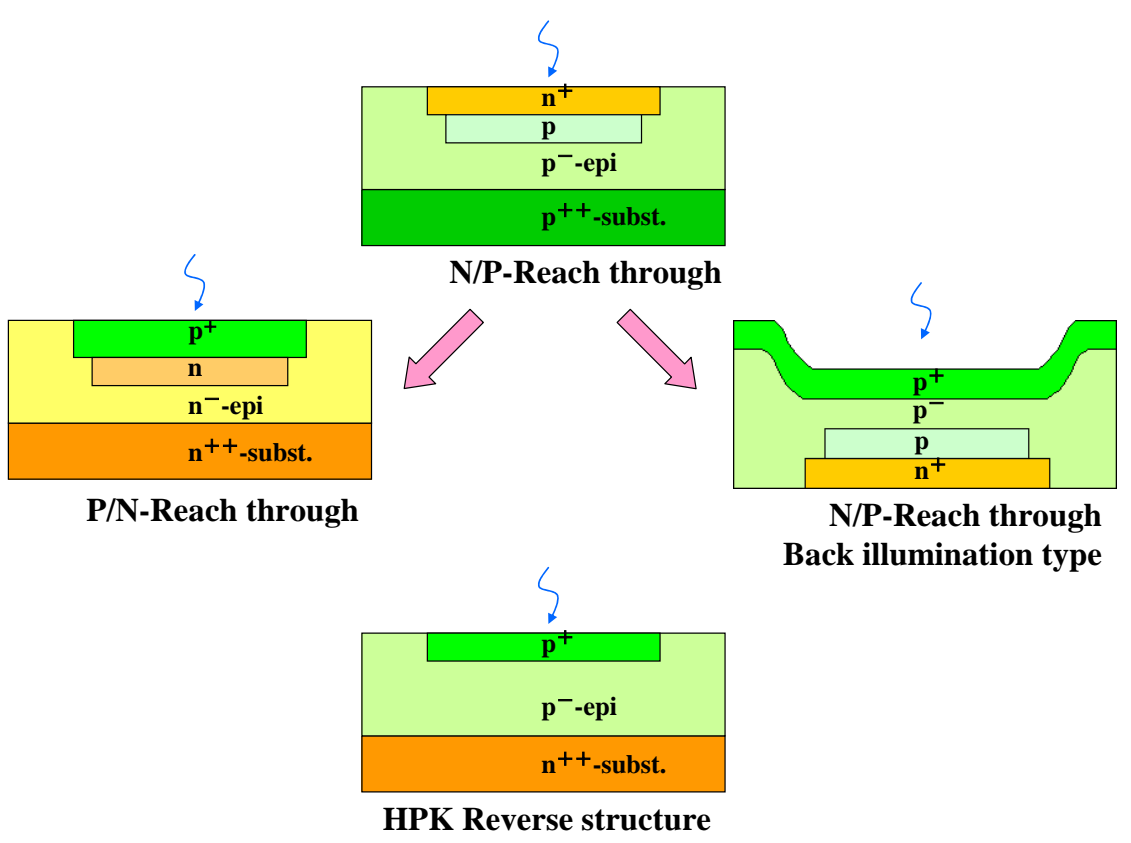

Fig. 7: The selection of MPPC structure. 


\section{Characteristics of MPPC}

\subsection{Gain, Dark count rate and PDE}

The main parameter that determine the characteristic of MPPC are gain, dark count rate and PDE. Fig. 8, 9 shows the gain and dark count rate of 400 and 100 pixel type MPPC respectively as a function of bias voltage. The PDE indicates in Fig. 10 shows that the peak sensitive wavelength is tuned to have around $400 \mathrm{~nm}$. This data has been taken by measuring the current mode, so the effect of after-pulse and cross-talk are included. These effects are not negligible and necessary to decrease in future. Fig. 11 shows the total specification of Hamamatsu S10362-11 series.

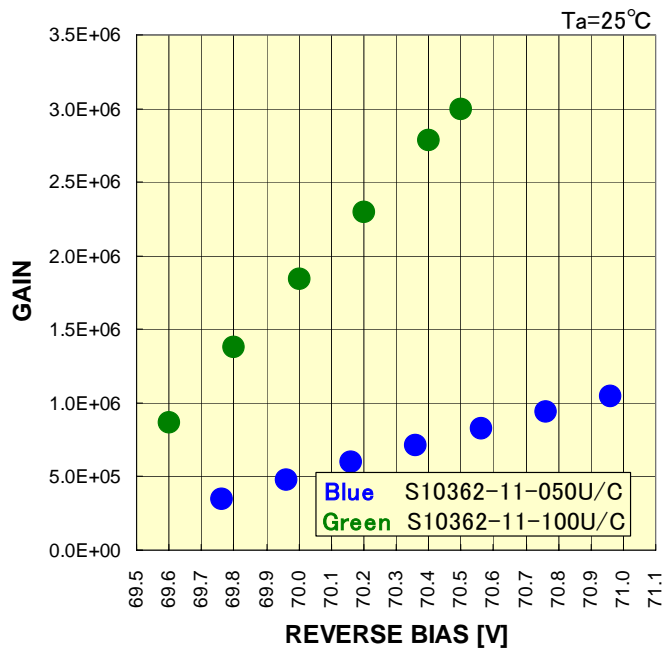

Fig. 8: gain vs. bias voltage

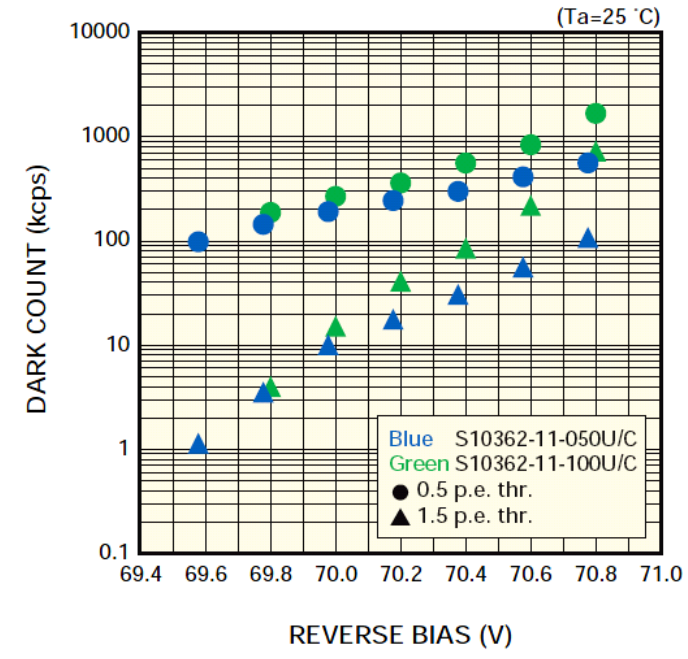

Fig. 9: dark count rate vs. bias voltage

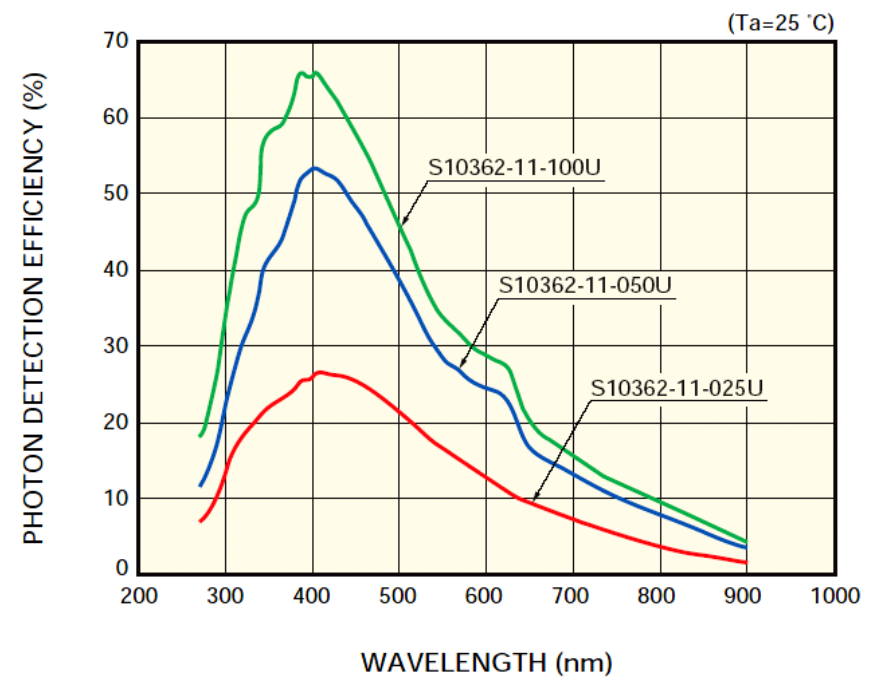

Fig. 10: PDE including after-pulse and cross-talk effect 
Specifications ( $\mathrm{Ta}=25^{\circ} \mathrm{C}$, Typ. unless otherwise noted)

\begin{tabular}{|c|c|c|c|c|c|}
\hline \multirow{2}{*}{ Parameter } & \multirow{2}{*}{ Symbol } & \multicolumn{3}{|c|}{ S10362-11 series } & \multirow{2}{*}{ Unit } \\
\hline & & $-025 \mathrm{U},-025 \mathrm{C}$ & $-050 \mathrm{U},-050 \mathrm{C}$ & $-100 \mathrm{U},-100 \mathrm{C}$ & \\
\hline Chip size & - & \multicolumn{3}{|c|}{$1.5 \times 1.5$} & $\mathrm{~mm}$ \\
\hline Effective active area & - & \multicolumn{3}{|c|}{$1 \times 1$} & $\mathrm{~mm}$ \\
\hline Number of pixels & - & 1600 & 400 & 100 & - \\
\hline Pixel size & - & $25 \times 25$ & $50 \times 50$ & $100 \times 100$ & $\mu \mathrm{m}$ \\
\hline Fill factor *1 & - & 30.8 & 61.5 & 78.5 & $\%$ \\
\hline Spectral response range & $\lambda$ & \multicolumn{3}{|c|}{270 to 900} & $\mathrm{~nm}$ \\
\hline Peak sensitivity wavelength & $\lambda p$ & \multicolumn{3}{|c|}{400} & $\mathrm{~nm}$ \\
\hline Quantum efficiency $(\lambda=\lambda p)$ & $\mathrm{QE}$ & \multicolumn{3}{|c|}{70 Min. } & $\%$ \\
\hline Photon detection efficiency ${ }^{{ }^{2}}(\lambda=\lambda p)$ & PDE & 25 & 50 & 65 & $\%$ \\
\hline Recommended operating voltage range & - & \multicolumn{3}{|c|}{$70 \pm 10^{\star 3}$} & $\mathrm{~V}$ \\
\hline Dark count & - & 100 & 270 & 400 & kcps \\
\hline Terminal capacitance & $\mathrm{Ct}$ & \multicolumn{3}{|c|}{35} & $\mathrm{pF}$ \\
\hline Time resolution (FWHM) & - & 250 & 220 & 250 & ps \\
\hline Temperature coefficient of reverse bias & - & \multicolumn{3}{|c|}{50} & $\mathrm{mV} /{ }^{\circ} \mathrm{C}$ \\
\hline Gain & $\mathrm{M}$ & $2.75 \times 10^{5}$ & $7.5 \times 10^{5}$ & $2.4 \times 10^{6}$ & - \\
\hline
\end{tabular}

*1: Ratio of the active area of a pixel to the entire area of the pixel.

*2: Photon detection efficiency includes crosstalk and afterpulses.

*3: For the recommended operating voltage of each product, refer to the data attached to each product.

Note: The last letter of each type number indicates package materials (U: metal, C: ceramic).

Fig. 11: Hamamatsu S10362-11 series specifications

\subsection{Output signal of MPPC}

The uniformity of each pixel can be seen to some extent by monitoring the output pulse. Fig. 12 shows the photon counting by pulse height and Fig. 13 shows the photon counting by output charge. The light is irradiating to whole pixels. Each figure shows the good separation of each numerical photon. This implies that each pixel have good uniformity for the whole MPPC active area.

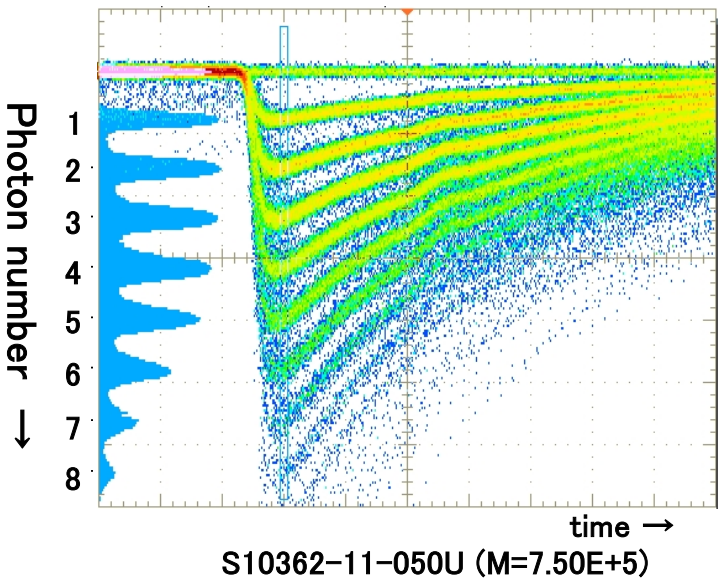

Fig. 12: Photon counting by pulse height (linear Amp.)

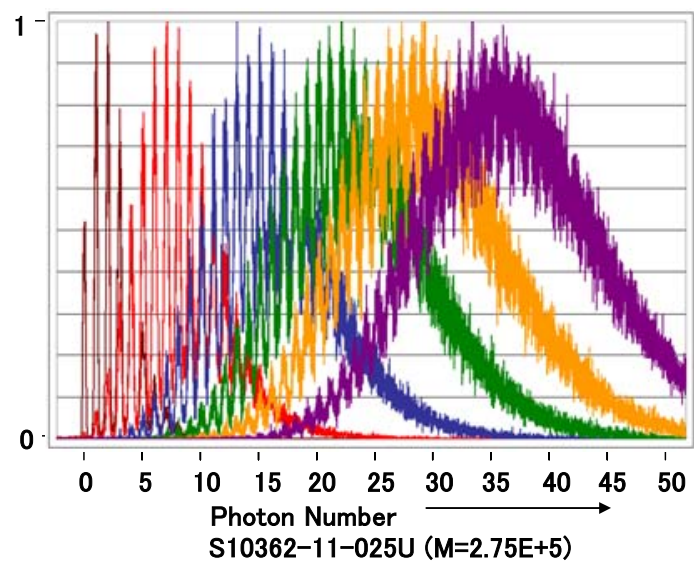

Fig. 13: Photon counting by output charge (charge Amp.)

\subsection{Time resolution}

In the region of time sensitive application like time-of-flight measurement, the existence of pulse jitter determine the performance of instrument. We estimated the pulse jitter of MPPC by Time Transition Spread (T.T.S.) method with one photoelectron threshold. Fig. 14 shows the 
full width of half maximum (FWHM) and full width at tenth maximum (FWTM) of jitter distribution (time resolution) in the case of irradiating to the center of pixel of 400 pixel type MPPC. There are not so many differences between pixels. Fig. 15 shows the position dependence of time resolution. Time resolution can be improved by applying higher bias voltage to MPPC in exchange for increase of darkcount, cross-talk and after-pulse.

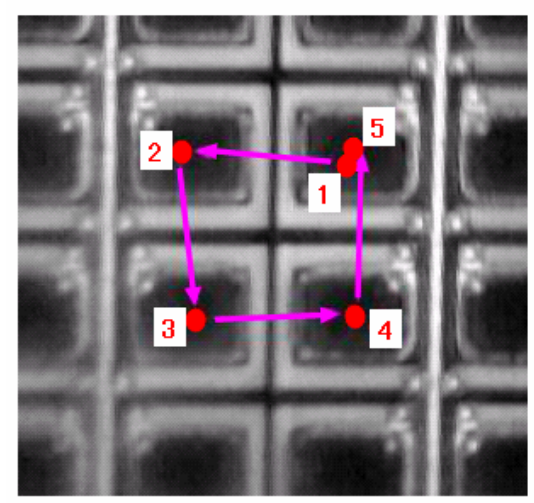

\begin{tabular}{|c|c|c|}
\hline & FWHM (ps) & FWTM (ps) \\
\hline 1 & 199 & 393 \\
\hline 2 & 197 & 389 \\
\hline 3 & 209 & 409 \\
\hline 4 & 201 & 393 \\
\hline 5 & 195 & 383 \\
\hline
\end{tabular}

Fig. 14: Time resolution at center $\left(1 \mathrm{~mm}^{2}, 50 \mathrm{um}\right.$ pitch)

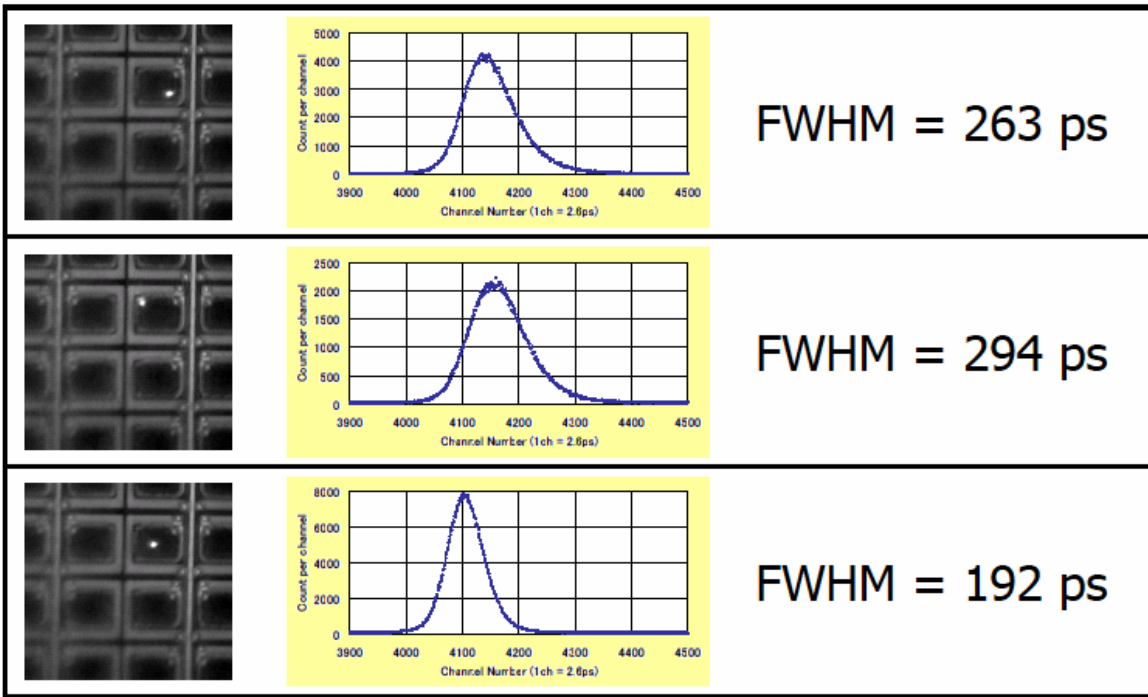

Fig. 15: Time resolution in one pixel $\left(1 \mathrm{~mm}^{2}, 50 u m\right.$ pitch)

\section{MPPC's future plan}

\subsection{Package development}

We have a plan on new packages. A small package enables to shrink the dead area when the plural number is tiled. It is also effective to shorten the active area and package surface length to improve the light collection efficiency. Fig. 16 shows some packages that we are developing. 


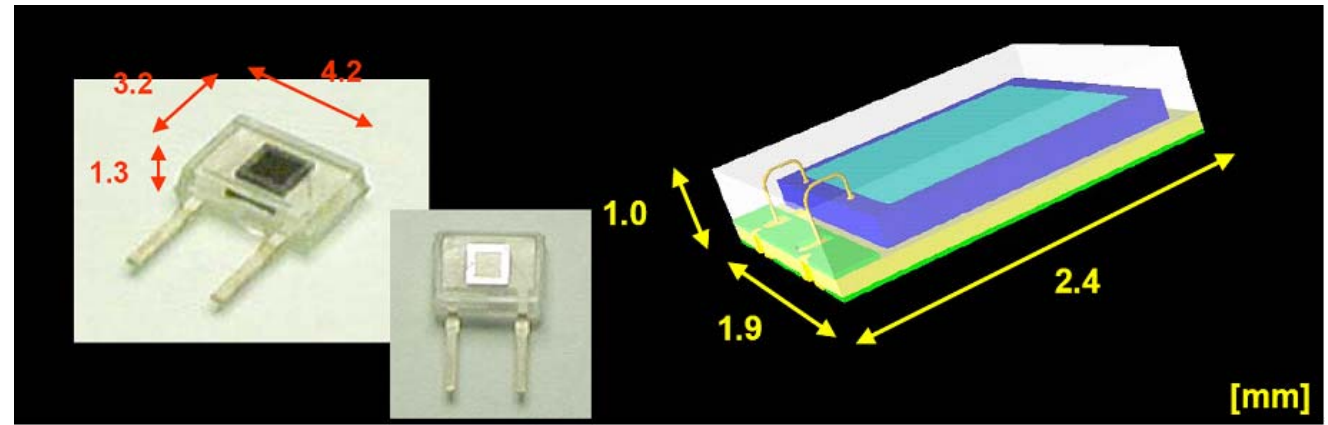

Fig. 16: Plastic PKG (left) and SMD PKG (right) for $1 \mathrm{~mm}^{2}$ MPPC (The sizes are not fixed)

\subsection{Large sensitive area, Array and Matrix}

From the viewpoint of dynamicrange and simplification of readout electronics, large area type and matrix type MPPC has a merit. We are developing $3 \mathrm{~mm}^{2}$ active area MPPC. The dark count rate is almost proportional to the active area. It implies that the dark count rate of the nine times larger MPPC reaches to few MHz. But the dark count signal height is still only a few photo-electron level. This means that the dark count are negligible in many case if the incident photon were high enough and if one set the appropriate threshold. Fig. 17 shows comparison of active area between $1 \mathrm{~mm}^{2}, 3 \mathrm{~mm}^{2}$ and $5 \mathrm{~mm}^{2}$ MPPC.

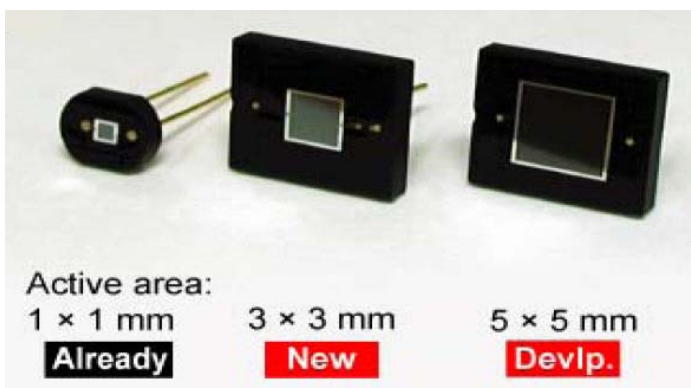

Fig. 17: Developing large active are type MPPC (Package is not fixed)

Fig. 18 shows the enegy resolution of $3 \mathrm{~mm}^{2}$ type MPPC (50um pitch, 3600 pixels) coupled with the LSO scintillator ( $3 \mathrm{~mm} \times 3 \mathrm{~mm} \times 20 \mathrm{~mm}$ ). The FWHM at $511 \mathrm{keV}$ could achieve $13 \%$. 


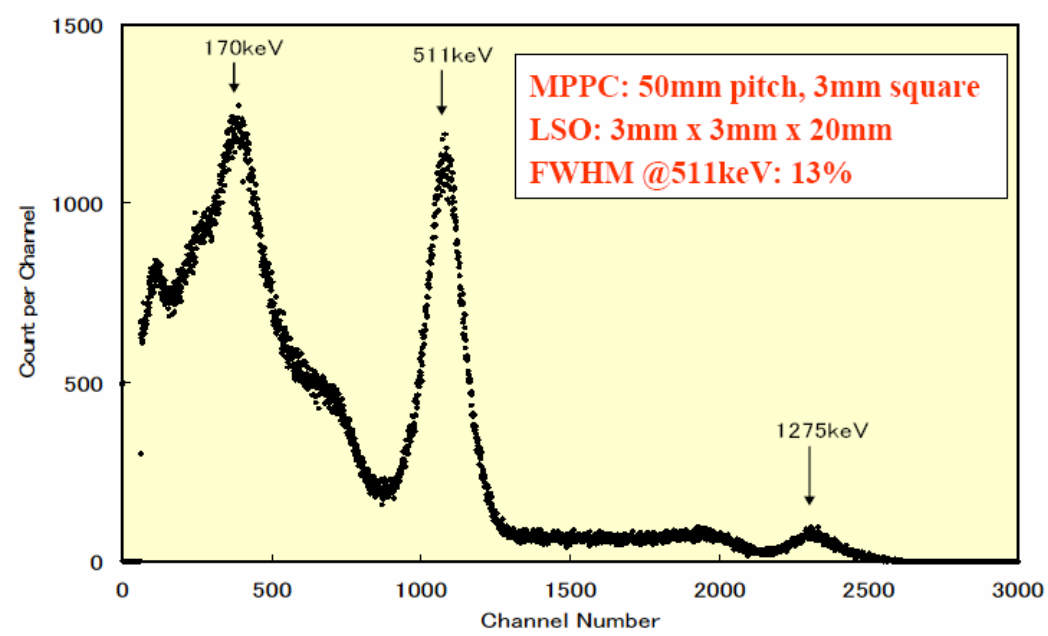

Fig. 18: Energy resolution measured by $3 \mathrm{~mm}^{2}$ MPPC coupled with LSO scintillator.

\subsection{Other development}

The other development we are planning are listed below.
a) Enhance PDE
b) Suppress after-pulse and cross-talk
c) Quenching resistor optimization
d) Package development (small, cheap, rugged)
e) Custom design (pitch, package, array)

One of ideas to suppress the optical cross-talk is making the trench etching between the pixels.

\section{MPPC Module}

There are MPPC module lineup for the fluorescence lifetime measurement, biological flow cytometry, bioluminescence analysis, ultralow light detection and analysis equipment (Fig. 19). MPPC module includes the amplifier, comparator, counter etc., so it's also convenient for the first evaluation of MPPC. There are two kinds of MPPC modules(1600pixel type and 400pixel type). The features of the MPPC module are listed below.

- Employs a MPPC

- Integrates a signal readout circuit ideal for MPPC

- Built-in high-voltage circuit and temperature-compensated circuit

- Three types of output : analog, comparator, pulse calculation value

- USB interface for easy handling : driven by USB bus power

- Compact and light weight 


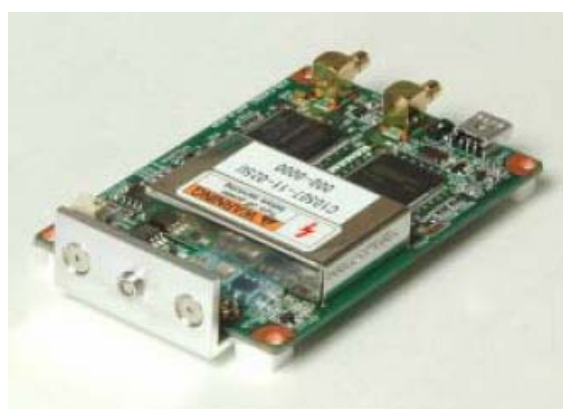

Fig. 19: Hamamastu MPPC module (C10507-11 series)

Fig. 20 and Fig. 21 show the MPPC module block diagram and analog output characteristics respectively.

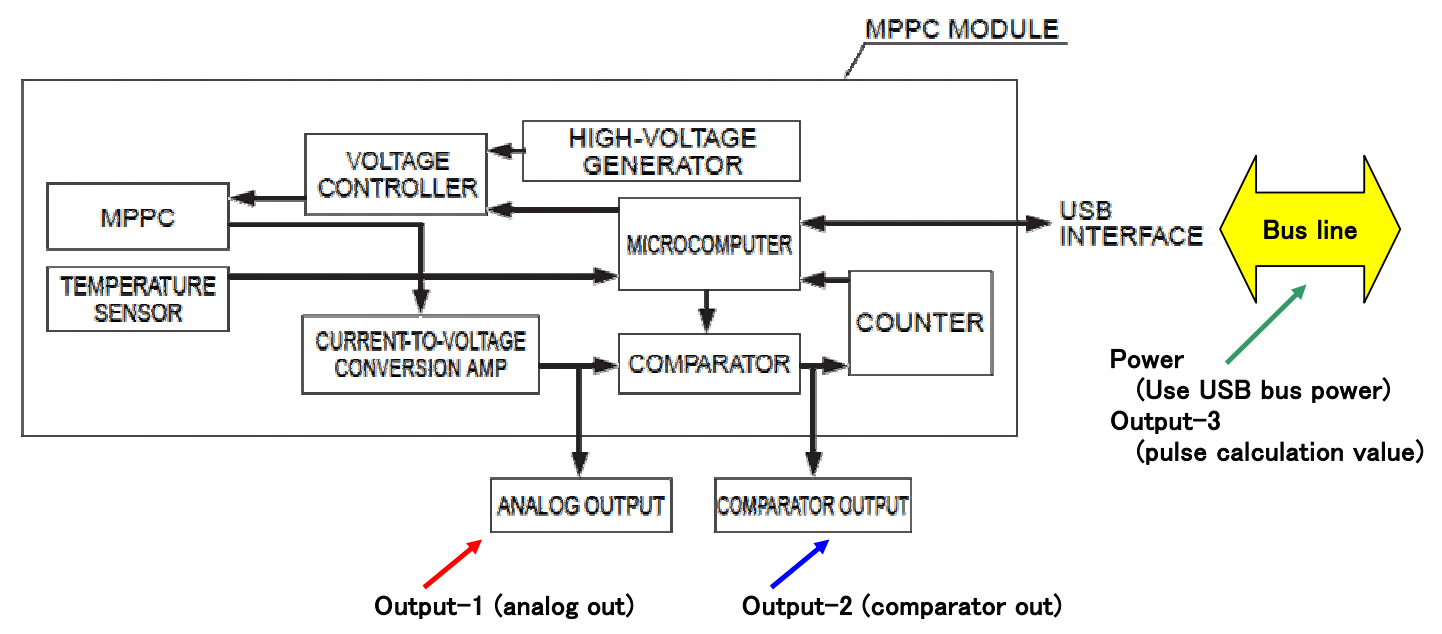

Fig. 20: MPPC module block diagram

\section{Analog output (C10507-11-025U)}

\section{Analog output (C10507-11-050U)}

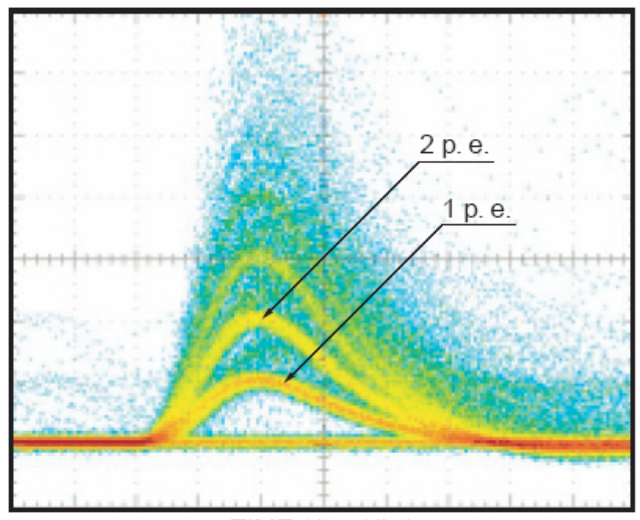

TIME (4 ns/div.)

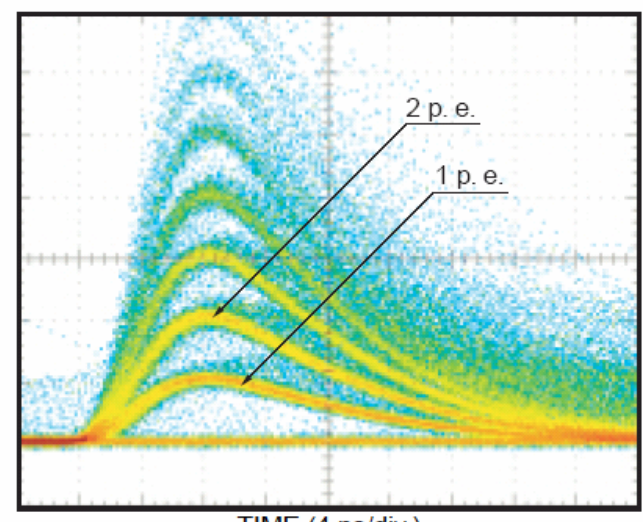

TIME (4 ns/div.)

Fig. 21: Analog output of MPPC module 


\section{Conclusion}

We fabricated silicon based photon counting device named MPPC, which is controlled to have peak sensitive wavelength around $400 \mathrm{~nm}$. The ability to distinguish the number of the photons, time resolution and PDE measurement indicate promising data. The smallness, robustness and low voltage operation will make handling easy. We hope new application regions will be explored by using new photon counting device.

\section{References}

[1] V. Golovin, V. Saveliev, "Novel type of avalanche photodetector with Geiger mode opration”, NIM A 518 (2004) 560

[2] V. Saveliev, “The recent development and study of silicon photomultiplier”, NIM A 535 (2004) 528

[3] A. N. Otte et al., "Prospects of Using Silicon Photomultipliers for the Astroparticle Physics Experiments EUSO and MAGIC“, IEE Trans. Nucl. Sci. 53 (2006) 636

[4] I. Britvitch et al., “Avalanche photodiodes now and possible developments”, NIM A 535 (2004) 523

[5] P. BUZHAN et al., "THE ADVANCED STUDY OF SILICON PHOTOMULTIPLIER”, Instrumentation Bulletin, Fall 2001, http://www.slac.stanford.edu/pubs/icfa/fall01.html

[6] Josef Šíp, “Quenching circuits for Single Photon Avalanche Photodiodes”, Proceedings of $8^{\text {th }}$ conference Student EEICT 2002, Volume 3, VUT Brno FEEC 2002

[7] W. C. Dash and R. Newman, "Intrinsic Optical Absorption in Single-Crystal Germanium and Silicon at $77^{\circ} \mathrm{K}$ and $300^{\circ} \mathrm{K}$ ” Phys. Rev., 99, 1151 (1955)

[8] Lee, C. A., R. A. Logan, R. L. Batdorf, J. J. Kleimack and W. Wiegmann, "Ionization Coefficients Measured in Abrupt InP Junctions“, Appl. Phys. Lett., 36, 4, p.302 (1980) 